\title{
ARTIGO ORIGINAL Prevalência de depressão e fatores associados em mulheres atendidas pela Estratégia de Saúde da Família
}

\author{
Prevalence of depression and associated factors in \\ women covered by Family Health Strategy
}

Angela Maria Corrêa Gonçalves', Maria Teresa Bustamante Teixeira', Jairo Roberto de Almeida Gama', Claudia Souza Lopes², Gulnar Azevedo e Silva³, Carmen Justina Gamarra², Kristiane de Castro Dias Duque', Maria Lucia Salim Miranda Machado ${ }^{4}$

\section{RESUMO}

Objetivo: Avaliar a prevalência de depressão e os fatores associados em mulheres de 20 a 59 anos de áreas cobertas pela Estratégia de Saúde da Família de município da Zona da Mata Mineira. Métodos: Trata-se de um estudo transversal, com mulheres de 20 a 59 anos cadastradas em duas Unidades de Saúde da Família, que utilizou um questionário contendo variáveis sociodemográficas, apoio social, autoavaliação de estado de saúde, estilo de vida, morbidade e saúde da mulher. O desfecho depressão foi avaliado segundo o Patients Health Questionnaire-9 (PHQ-9). Resultados: Das 1.958 mulheres incluídas nesta análise, 28,5\% encontram-se na faixa etária entre 30 e 39 anos; 15,4\% não concluíram o ensino elementar; $54,5 \%$ não trabalham ou nunca trabalharam; $44,2 \%$ declararam não ser da raça branca. Fatores associados à ocorrência de depressão na população estudada: possuir baixa escolaridade, trabalhar atualmente e ter doença mental prévia. Como fatores de proteção observaram-se: ser casada ou viver com companheiro, realizar atividades físicas regularmente e relatar autoavaliação positiva de saúde. Conclusão: Os resultados deste estudo revelam prevalência de depressão de 19,7\% nas mulheres de 20 a 59 anos de áreas cobertas pela Estratégia de Saúde

\section{Palavras-chave \\ Depressão, fatores associados, atenção primária à saúde, prevalência.} da Família, apontando para a necessidade de um cuidado especial na atenção primária à saúde às mulheres com baixa escolaridade, que trabalham, apresentam doença mental e não praticam exercícios físicos, de modo que se possa reduzir o sofrimento e promover a saúde. Ressalta-se a lacuna na utilização de instrumentos de rastreamento dos casos de depressão na atenção primária.

\begin{abstract}
Objective: To assess the prevalence of depression and its associated factors in women aged 20 to 59 years at some areas with the coverage of Family Health Strategy in a city located at Zona da Mata Mineira. Methods: It is a cross-sectional study with women aged 20-59 enrolled in two Primary Health Care Units making use of a questionnaire containing socio-
\end{abstract}

1 Universidade Federal de Juiz de Fora (UFJF), Juiz de Fora, MG, Brasil. 2 Universidade Federal da Integração Latino-Americana de Foz de Iguaçu (Unila), Foz do Iguaçu, PR, Brasil. 3 Universidade do Estado do Rio de Janeiro (UERJ), Rio de Janeiro, RJ, Brasil. 


\section{Keywords}

Depression, associated

factors, primary health care, prevalence. demographic variables, social support, self-assessment of general health status, lifestyle, morbidity and women's health. Depression was evaluated according to Patients Health Questionnaire-9 (PHQ-9). Results: From 1,958 women included in this analysis, 28,5\% are aged 30-39; 15,4\% did not finish High School; 54,5\% do not work or have never worked and $44,2 \%$ defined themselves as not white. Factors associated with depression episodes in the population studied: lower educational background, currently having a job, previous diagnosis of psychiatric disorders. Protective factors which were observed: being married or living with a partner, practicing physical activities in a regular basis and reporting positive selfassessed health. Conclusion: The results of this study reveal a prevalence of depression of $19.7 \%$ in women aged 20 to 59 years covered by the Family Health Strategy, pointing to the need for special care in primary health care for women with low schooling, who work, have mental illness, and do not exercise so that they can reduce suffering and promote health. It was observed a significant gap related to managing specific tools designed to screening depressive episodes in primary care.

\section{INTRODUÇÃO}

Os transtornos depressivos constituem um grave problema de saúde pública devido a sua alta prevalência, repercussões na saúde geral e impacto psicossocial. Segundo o Diagnostic and Statistical Manual of Mental Disorders (DSM-V)', são critérios para diagnóstico de depressão: estado deprimido (sentir-se deprimido a maior parte do tempo); anedonia: interesse diminuído ou perda de prazer para realizar as atividades de rotina; sensação de inutilidade ou culpa excessiva; dificuldade de concentração: habilidade frequentemente diminuída para pensar e concentrar-se; fadiga ou perda de energia; distúrbios do sono: insônia ou hipersonia praticamente diárias; problemas psicomotores: agitação ou retardo psicomotor; perda ou ganho significativo de peso, na ausência de regime alimentar; ideias recorrentes de morte ou suicídio ${ }^{2}$. Conforme o número de itens respondidos afirmativamente, o estado depressivo pode ser classificado em três grupos: a) depressão menor: dois a quatro sintomas por duas ou mais semanas, incluindo estado deprimido ou anedonia; b) distimia: três ou quatro sintomas, incluindo estado deprimido, durante dois anos, no mínimo; c) depressão maior: cinco ou mais sintomas por duas semanas ou mais, incluindo estado deprimido ou anedonia.

Pacientes deprimidos são frequentadores assíduos de serviços de atendimento primário, porém, muitas vezes, não são diagnosticados como tal'. Um Relatório da Organização Mundial de Saúde (OMS) aponta que o número de casos de depressão aumentou 18\% entre 2005 e 2015: são 322 milhões de pessoas em todo o mundo, a maioria mulheres. No Brasil, a depressão atinge 11,5 milhões de pessoas $(5,8 \%$ da população) $)^{3}$.

A literatura demonstra que "os índices de depressão na atenção primária à saúde apresentam frequências consideráveis tanto nos estudos nacionais quanto nos estudos internacionais" ${ }^{\prime \prime}$, contudo observam-se maiores índices nos estudos desenvolvidos no Brasil, o que aponta lacunas na assistência e nas políticas públicas e pouco investimento em pesquisas nesse âmbito ${ }^{5-11}$.

No Brasil, uma pesquisa populacional de 2013 com amostra nacionalmente representativa, a Pesquisa Nacional de Saúde (PNS), mostrou que a prevalência de depressão maior é mais significativa entre as mulheres que vivem em áreas urbanas, com níveis educacionais mais baixos e com condições crônicas, como hipertensão e diabetes ${ }^{12}$. Dados da mesma pesquisa evidenciaram inequidades no acesso aos cuidados em saúde mental. Verificou-se que a maioria dos brasileiros com sintomas depressivos clinicamente relevantes $(78,8 \%)$ não recebia nenhum tipo de tratamento ${ }^{12}$.

A depressão se apresenta como uma das principais causas de incapacitação no mundo, limitando o funcionamento físico, pessoal e social. Em ambulatórios de Atenção Primária, a prevalência de depressão foi estimada em 5\% a 10\% de todos os pacientes, e apenas um em cem cita a doença como motivo da consulta, e em até 50\% das vezes o problema não é detectado pelos profissionais ${ }^{4}$. Estudos estabeleceram correlação entre estado depressivo e piora de quadros clínicos, tais como cardiopatias, diabetes, obesidade e problemas oncológicos ${ }^{2,12}$, e ainda associação significativa entre depressão na maternidade, problemas no desenvolvimento infantil e piora no rendimento escolar, com implicações para o ambiente familiar ${ }^{13}$.

Diversos instrumentos de screening para detecção de depressão na população foram desenvolvidos, como: World Health Organization Quality of Life (WHOQOL-bref), Mental Health Index 5 (MHI-5), Centers for Epidemiologic Studies - Depression (CES-D), sendo o mais utilizado deles o Patient Health Questionnaire (PHQ-9) da Associação Psiquiátrica Americana (APA) ${ }^{14,15}$. Esses instrumentos têm sido avaliados como um avanço na detecção de possíveis casos de depressão na população, dada sua sensibilidade e confiabilidade, podendo tanto ser aplicados por entrevistadores treinados como ser autoadministrados. 
Busca-se com este estudo avaliar a prevalência de depressão e os fatores associados em mulheres de 20 a 59 anos de áreas cobertas pela Estratégia de Saúde da Família do município de Juiz de Fora-MG.

\section{MÉTODOS}

Este estudo é parte da pesquisa "Avaliação de estratégias para rastreamento do câncer de colo do útero em mulheres cobertas pela Estratégia Saúde da Família (ESF) do município de Juiz de Fora (MG)". Trata-se de um estudo transversal com captação domiciliar e coleta de dados realizada em duas Unidades de Atenção Primária à Saúde (UAPS), no município de Juiz de Fora, Minas Gerais, em mulheres de 20 a 59 anos, faixa etária alvo para o rastreamento citopatológico, excluindo-se as grávidas, virgens e histerectomizadas.

Foram identificadas, a partir de levantamento realizado pelos Agentes Comunitários de Saúde, 4.516 mulheres nessa faixa etária, residentes nas áreas adscritas. A captação das mulheres para participação da pesquisa se deu por meio de convite no domicílio pelos agentes e, no caso de não comparecimento, foram realizadas outras duas tentativas de agendamento, por meio de nova visita domiciliar e/ou contato telefônico. Participaram do estudo 2.077 mulheres, o que correspondeu a $46 \%$ do universo. Para a presente análise, foram consideradas as 1.958 mulheres que responderam ao PHQ-9.

A coleta de dados foi realizada entre julho de 2011 e junho de 2012 por meio de um questionário adaptado do instrumento utilizado na Pesquisa Nacional de Saúde ${ }^{16,17}$, após a autorização de seus coordenadores. Todos os participantes assinaram o termo de consentimento livre e esclarecido.

O questionário foi aplicado por profissionais de nível superior - previamente capacitados -, em local reservado e individualizado, antes da realização do exame ginecológico. Os dados foram armazenados em um banco desenvolvido no programa Epi Info (versão 6.04b).

O desfecho depressão foi avaliado por meio da versão brasileira do Questionário de Saúde do Paciente (PHQ-9) ${ }^{14}$, já validado no Brasil. Tal questionário é composto por nove questões que avaliam a frequência de sintomas depressivos nos últimos 15 dias, baseado nos critérios para o diagnóstico de depressão do Manual de Diagnóstico e Estatística de Transtornos Mentais (DSM-IV) ${ }^{18}$. Apresenta quatro possibilidades de resposta para cada item, que definem as respectivas pontuações: nenhuma vez (0); vários dias (1); mais da meta-

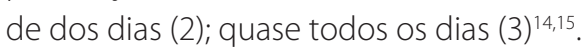

O PHQ-9 pode ser interpretado sob a forma de algoritmo, no rastreamento e identificação de indivíduos com episódios depressivos, seja como uma medida contínua, com escores de 0 a 27 pontos, avaliando os níveis de sintomas de depressão por meio dos pontos de corte (superior ou igual a 5, 10, 15 e 20 pontos, representando, respectivamente, de- pressão leve, moderada, severa e grave), seja classificando os indivíduos de modo dicotômico ${ }^{15}$.

No presente estudo, foi utilizado o ponto de corte maior ou igual a 10 para depressão, que, em estudo de validação com população de adultos de cidade de médio porte no sul do Brasil, revelou sensibilidade de 72,5\% (IC 95\%: 61,5\% a 89,2\%) e especificidade de 88,9\% (IC 95\%: 83,0\% a 89,9\%) $)^{14,15}$.

Foram consideradas como positivas para depressão as mulheres que, no questionário PHQ-9, obtiveram escore maior ou igual a 10 pontos, que consistiu na variável dependente do estudo. Quando detectado o transtorno, a voluntária foi encaminhada para fazer acompanhamento gratuitamente.

As variáveis independentes foram agrupadas em variáveis sociodemográficas (idade, cor da pele autorreferida, situação conjugal, escolaridade, ter religião, trabalho, renda mensal per capita, apoio social); de estilo de vida (consumo de bebida alcoólica, uso de tabaco e prática regular de atividade física); de situação de saúde (autoavaliação do estado de saúde, doença mental, uso de medicamento para dormir, atendimento na UAPS).

A análise desenvolveu-se com os seguintes passos: análise univariada para a descrição da distribuição das variáveis dependentes e independentes na população estudada; análise bivariada para identificação de associação de cada uma das variáveis independentes com a variável dependente, dicotômica, sendo calculadas as razões de prevalência brutas $\left(\mathrm{RP}_{\text {Bruta }}\right)$ com os respectivos intervalos de confiança de $95 \%$ (IC 95\%) e valores de $p$. Para a análise multivariada, por meio de regressão robusta de Poisson, foram selecionadas as variáveis que apresentaram valor de $p<0,20$ na análise bivariada. Utilizou-se para tal o programa estatístico Stata 11.0 (StataCorp. College Station, Estados Unidos).

O presente estudo foi aprovado pela Comissão de Ética (CEP) do Instituto de Medicina Social (IMS) da Universidade do Estado do Rio de Janeiro (UERJ), conforme parecer no 0026.1.259.180-09. A pesquisa foi submetida à avaliação do CEP da Universidade Federal de Juiz de Fora e aprovada sob o parecer n 068/2011.

\section{RESULTADOS}

Entre 1.958 mulheres avaliadas pelo PHQ-9, 387 pontuaram como positivas para depressão, o que corresponde a uma prevalência de 19,7\%. Cabe ressaltar que, entre as $387 \mathrm{mu}$ Iheres que obtiveram pontuação maior ou igual a 10, 205 (53\%) não relataram tal transtorno quando questionadas diretamente no item morbidade do instrumento da pesquisa.

Na Tabela 1, verifica-se que a presença de depressão mostrou associação com fatores como escolaridade, situação conjugal e de trabalho, renda mensal, apoio social de parentes, autoavaliação de saúde, prática regular de atividade física, tabagismo, doença mental, uso de medicamentos para dormir e procura por atendimento na UAPS no último ano. 
Tabela 1. Descrição da amostra, prevalência de depressão e fatores associados em mulheres de 20 a 59 anos, assistidas pela ESF do município de Juiz de Fora (MG)

\begin{tabular}{|c|c|c|c|c|c|c|c|}
\hline \multirow{2}{*}{ Variáveis } & \multicolumn{2}{|c|}{ Total* } & \multirow[b]{2}{*}{ P Depressão } & \multirow[b]{2}{*}{$\%$} & \multirow{2}{*}{ RPBruta } & \multirow{2}{*}{ IC95\% } & \multirow{2}{*}{$P$} \\
\hline & N & $\%$ & & & & & \\
\hline Total & 1.958 & 100,0 & 387 & 19,77 & & & \\
\hline \multicolumn{8}{|l|}{ Idade (anos) } \\
\hline $20-29$ & 517 & 26,4 & 100 & 19.3 & 1 & & \\
\hline $30-39$ & 559 & 28,5 & 109 & 19,5 & 1,00 & $0,79-1,28$ & 0,94 \\
\hline $40-49$ & 485 & 24,8 & 89 & 18,3 & 0,94 & $0,73-1,22$ & 0,68 \\
\hline $50-59$ & 397 & 20,3 & 89 & 22,4 & 1,15 & $0,89-1,49$ & 0,25 \\
\hline \multicolumn{8}{|l|}{ Escolaridade } \\
\hline Elementar completo/EM & 1.644 & 84,6 & 308 & 18,7 & 1 & & \\
\hline Até elementar incompleto & 299 & 15,4 & 79 & 26,4 & 1,41 & $1,14-1,75$ & 0,002 \\
\hline \multicolumn{8}{|l|}{ Cor/raça } \\
\hline Branca & 1.092 & 55,8 & 210 & 19,2 & 1 & & \\
\hline Outras & 866 & 44,2 & 177 & 20,4 & 1,06 & $0,88-1,27$ & 0,505 \\
\hline \multicolumn{8}{|l|}{ Ter religião } \\
\hline Sim & 1.897 & 98,3 & 369 & 19,4 & 1 & & \\
\hline Não & 33 & 1,7 & 8 & 24,2 & 1,24 & $0,68-2,29$ & 0,479 \\
\hline \multicolumn{8}{|l|}{ Situação conjugal } \\
\hline Solteira e outros & 758 & 38,7 & 176 & 23,2 & 1 & & \\
\hline Casada ou com companheiro & 1.199 & 61,3 & 211 & 17,6 & 0,75 & $0,63-0,91$ & 0,002 \\
\hline \multicolumn{8}{|l|}{ Situação de trabalho } \\
\hline Não trabalha/nunca trabalhou & 1.065 & 54,5 & 170 & 16,0 & 1 & & \\
\hline Trabalha atualmente & 890 & 45,5 & 217 & 24,4 & 1,52 & $1,28-1,82$ & $<0,001$ \\
\hline \multicolumn{8}{|l|}{ Renda mensal (per capita) } \\
\hline Média e alta & 1.227 & 62,7 & 220 & 18,0 & 1 & & \\
\hline Baixa & 731 & 37,3 & 167 & 22,8 & 1,27 & $1,07-1,52$ & 0,008 \\
\hline \multicolumn{8}{|l|}{ № de pessoas na residência } \\
\hline Uma ou menos & 62 & 3,2 & 16 & 25,8 & 1 & & \\
\hline Duas ou mais & 1.894 & 96,8 & 371 & 19,6 & 0,75 & $0,49-1,16$ & 0,21 \\
\hline \multicolumn{8}{|l|}{ Apoio de parentes } \\
\hline Sem apoio & 488 & 25,0 & 120 & 24,6 & 1 & & \\
\hline Com apoio & 1.463 & 75,0 & 264 & 18,0 & 0,73 & $0,60-0,88$ & 0,001 \\
\hline \multicolumn{8}{|l|}{ Apoio de amigos } \\
\hline Sem apoio & 857 & 44,0 & 182 & 21,2 & 1 & & \\
\hline Com apoio & 1.092 & 56,0 & 201 & 18,4 & 0,86 & $0,72-1,03$ & 0,118 \\
\hline \multicolumn{8}{|l|}{ Autoavaliação de saúde } \\
\hline Regular, ruim e muito ruim & 812 & 41,6 & 259 & 31,9 & 1 & & \\
\hline Muito boa e boa & 1.139 & 58,4 & 124 & 10,9 & 0,34 & $0,28-0.41$ & $<0,001$ \\
\hline \multicolumn{8}{|l|}{ Prática regular de atividade física } \\
\hline Sim & 444 & 22,7 & 66 & 14,9 & 1 & & \\
\hline Não & 1.513 & 77,3 & 321 & 21,2 & 0,70 & $0,55-0,89$ & 0,004 \\
\hline \multicolumn{8}{|l|}{ Consumo de álcool } \\
\hline Sim & 837 & 42,8 & 171 & 20,4 & 1 & & \\
\hline Não & 1.120 & 57,2 & 216 & 19,3 & 0,94 & $0,78-1,12$ & 0,52 \\
\hline \multicolumn{8}{|l|}{ Tabagismo } \\
\hline Não & 1.531 & 78,7 & 281 & 18,3 & 1 & & \\
\hline Sim & 415 & 21,3 & 101 & 24,3 & 1,32 & $1,09-1,62$ & 0,006 \\
\hline
\end{tabular}




\begin{tabular}{|c|c|c|c|c|c|c|c|}
\hline \multirow{2}{*}{ Variáveis } & \multicolumn{2}{|c|}{ Total* } & \multirow[b]{2}{*}{ PDepressão } & \multirow[b]{2}{*}{$\%$} & \multirow{2}{*}{ RPBruta } & \multirow{2}{*}{ IC $95 \%$} & \multirow{2}{*}{$\mathbf{P}$} \\
\hline & N & $\%$ & & & & & \\
\hline \multicolumn{8}{|c|}{ Frequência a cultos } \\
\hline Não & 560 & 28,7 & 121 & 21,6 & 1 & & \\
\hline $\operatorname{Sim}$ & 1.390 & 71,3 & 262 & 18,8 & 0,87 & $0,72-1,05$ & 0,163 \\
\hline \multicolumn{8}{|c|}{ Apresenta doença mental (esquizofrenia, transtorno bipolar, psicoses) } \\
\hline Não & 1.905 & 97,3 & 362 & 19,0 & 1 & & \\
\hline $\operatorname{sim}$ & 53 & 2,7 & 25 & 47,2 & 2,48 & $1,84-3,35$ & $<0,001$ \\
\hline \multicolumn{8}{|c|}{ Uso de medicamento para dormir } \\
\hline Não & 1.024 & 79,2 & 187 & 18,3 & 1 & & \\
\hline Sim & 269 & 20,8 & 127 & 47,2 & 2,59 & $2,16-3,10$ & $<0,001$ \\
\hline \multicolumn{8}{|c|}{ Procurou atendimento na UAPS } \\
\hline $\operatorname{Sim}$ & 757 & 49,0 & 119 & 15,7 & 1 & & \\
\hline Não & 786 & 59,1 & 173 & 22,0 & 1,40 & $1,13-1,73$ & 0,002 \\
\hline
\end{tabular}

* 0 total (n) de cada variável pode diferir devido à ocorrência de dados ignorados.

A prevalência do evento foi maior $(22,42 \%)$ na faixa etária de 50 a 59 anos, embora a idade não tenha apresentado associação significante com o desfecho. Possuir menor escolaridade (RP: 1,41; IC 95\%: 1,14-1,75), trabalhar atualmente (RP: 1,52; IC 95\%: 1,28-1,82), ter baixa renda (RP: 1,27; IC 95\%: 1,07-1,52), relatar doença mental (RP: 2,48; IC 95\%: 1,84-3,35), usar medicamento para dormir (RP: 2,59; IC 95\%: 2,16-3,10), ser tabagista (RP: 1,32; IC 95\%: 1,09-1,62) e ter procurado atendimento na UAPS no último ano (RP: 1,59; IC 95\%: 1,24$2,03)$ estiveram associados a maior risco de apresentar depressão segundo os critérios do PHQ-9; enquanto viver com companheiro (RP: 0,75; IC 95\%: 0,63-0,91), ter apoio social de parentes (RP: 0,73; IC 95\%: 0,60-0,88), praticar regularmente atividade física (RP: 0,70; IC 95\%: 0,55-0,89) e ter autoavaliação de saúde muito boa ou boa (RP: 0,34; IC 95\%: 0,28-0,41) constituíram-se fatores de proteção contra a depressão.

Na Tabela 2, são apresentados os resultados da análise multivariada ajustada por idade, que confirma a associação de estar trabalhando atualmente (RP: 1,34; IC 95\%: 1,12-1,61) e relatar doença mental (RP: 1,86; IC 95\%: 1,39-2,49) como fatores de risco para a depressão e limítrofe para menor escolaridade (RP: 1,23; IC 95\%: 0,99-1,53). Estar casada ou viver com companheiro (RP: 0,77; IC 95\%: 0,65-0,92), autoavaliar-se com saúde boa ou muito boa (RP: 0,37; IC 95\%: 0,30-0,45) e praticar atividade física (RP: 0,75; IC 95\%: 0,59-0,94) mostraram-se como fatores de proteção para essa condição.

Tabela 2. Razão de prevalência (bruta e ajustada) de depressão, segundo variáveis selecionadas, em mulheres de 20 a 59 anos assistidas pela ESF do município de Juiz de Fora (MG)

\begin{tabular}{|c|c|c|c|c|c|c|}
\hline Variáveis & RPbr & IC $95 \%$ & $P$ & RPaj* $^{*}$ & IC $95 \%$ & $P$ \\
\hline \multicolumn{7}{|l|}{ Escolaridade } \\
\hline Elementar completo/EM & 1 & & & 1 & & \\
\hline Até elementar incompleto & 1,41 & $1,14-1,75$ & 0,002 & 1,23 & $0,99-1,53$ & 0,058 \\
\hline \multicolumn{7}{|l|}{ Situação conjugal } \\
\hline Solteira e outros & 1 & & & 1 & & \\
\hline Casada ou com companheiro & 0,75 & $0,63-0,91$ & 0,002 & 0,77 & $0,65-0,92$ & 0,003 \\
\hline \multicolumn{7}{|l|}{ Situação de trabalho } \\
\hline Não trabalha/nunca trabalhou & 1 & & & 1 & & \\
\hline Trabalha atualmente & 1,52 & $1,28-1,82$ & $<0,001$ & 1,34 & $1,12-1,61$ & $<0,001$ \\
\hline \multicolumn{7}{|l|}{ Autoavaliação de saúde } \\
\hline Regular, ruim e muito ruim & 1 & & & 1 & & \\
\hline Muito boa e boa & 0,34 & $0,28-0,41$ & $<0,001$ & 0,37 & $0,30-0.45$ & $<0,001$ \\
\hline \multicolumn{7}{|l|}{ Prática regular de atividade física } \\
\hline Sim & 1 & & & 1 & & \\
\hline Não & 0,70 & $0,55-0,89$ & 0,004 & 0,75 & $0,59-0,94$ & 0,014 \\
\hline \multicolumn{7}{|c|}{ Apresenta doença mental (esquizofrenia, transtorno bipolar, psicoses) } \\
\hline Não & 1 & & & 1 & & \\
\hline Sim & 2,48 & $1,84-3,35$ & $<0,001$ & 1,86 & $1,39-2,49$ & $<0,001$ \\
\hline
\end{tabular}

* Ajustadas poridade. 


\section{DISCUSSÃO}

No presente estudo, a prevalência de depressão encontrada nas mulheres foi de $19,7 \%$, próximo ao valor encontrado em metanálise ${ }^{19}$ de estudos em adultos brasileiros que apurou uma prevalência de 17\% (IC 95\%: 14-19), sendo de 21,6\% (IC 95\%: 18,5-24,7) em mulheres e de 9,7\% (IC 95\%: 7,5-11,8) em homens. Em estudo transversal de base populacional que incluiu 1.593 adultos do Distrito Federal, dos quais 58,3\% eram mulheres, a depressão foi autorreferida por 11,2\% (IC 95\%: 9,812,9\%) dos entrevistados, sendo de 14,6\% (IC 95\%: 12,0-17,6\%) em mulheres e de 7,7\% (IC 95\%: 5,6-10,2) em homens ${ }^{20}$.

Vários estudos epidemiológicos sustentam a informação de que a depressão é aproximadamente duas vezes mais prevalente em mulheres do que em homens ${ }^{2,19-22}$. Os mesmos estudos têm buscado aferir os fatores explicativos para essa diferença e apontam como fatores relevantes as diferenças fisiológicas e hormonais, baixo nível de escolaridade, baixa renda, questões socioculturais, além de diferentes formas de lidar com situações estressoras.

Verificou-se, neste estudo, que as mulheres com cor da pele branca apresentaram maior prevalência de depressão em comparação as mulheres com cor da pele preta. Esse resultado contraria os de estudos que observaram maior prevalência de depressão nos indivíduos de cor da pele preta, em comparação com os de pele branca. Alguns estudos ressaltam que desigualdades em saúde não devem ser atribuídas, exclusivamente, à cor da pele, sendo necessário considerar, também, as desigualdades econômicas e sociais em diferentes contextos ${ }^{23,24}$.

No que se refere à situação conjugal, constatou-se que as mulheres solteiras ou sem companheiro apresentaram maior prevalência de depressão. O resultado obtido comun-

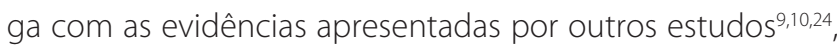
revelando que viver com companheiro e ter apoio social funciona como efeito protetor contra a depressão ${ }^{24,25}$.

No presente estudo, observou-se que $98 \%$ das mulheres afirmaram ter religião, no entanto não foi encontrada associação com aspectos religiosos ou frequência a cultos. Embora a religiosidade possa influenciar o modo como pessoas lidam com situações de estresse, sofrimento e problemas vitais, pode levar a um sentimento de culpa, dúvida e autocrítica, o que pode contribuir para a depressão ${ }^{24}$.

O consumo de bebidas alcoólicas referido nos últimos 30 dias foi comum a $42,8 \%$ das mulheres entrevistadas. $O$ abuso ou dependência do álcool costuma estar relacionado com a depressão, pois pode ser uma forma que o indivíduo encontra para atenuar os sintomas9. No entanto, neste estudo, não houve associação entre a depressão e o uso de álcool.

Quanto ao tabagismo, 21,3\% das entrevistadas referiram fazer uso diário de pelo menos um maço de cigarros. A depressão é a comorbidade psiquiátrica mais comumente associada à dependência de nicotina ${ }^{25}$. "Há forte evidência de comorbidade entre tabagismo e transtornos depressivos", e a cessação do tabagismo é fator de risco para a manutenção do quadro clínico ou o desenvolvimento de novo episódio depressivo nesses usuários, isto é, a relação entre tabagismo e depressão é bidirecional2 ${ }^{26}$. Embora estudos apresentem evidências de que há associação entre depressão e dependência nicotínica, isso não se confirmou neste estudo, quando ajustado pelas outras variáveis ${ }^{19,26}$.

O uso de "medicamento para dormir", neste estudo, foi relatado por $20,8 \%$ das entrevistadas e por $47,2 \%$ entre aquelas com depressão, bem abaixo do encontrado na literatura, que afirma que os transtornos do sono estão presentes em aproximadamente $80 \%$ dos casos de depressão e sua deterioração se dá tanto em qualidade quanto em quantidade ${ }^{27}$. As queixas incluem dificuldade para iniciar o sono, despertares noturnos, sono não restaurador, redução do sono total, sonhos perturbadores e despertar precoce pela manhã28.

Salienta-se que pessoas com sintomas depressivos costumam fazer mais visitas ao médico, são submetidas a maior quantidade de exames e usam mais medicações quando comparadas a pessoas sem depressão ${ }^{29}$. Neste estudo, observa-se que a ocorrência de depressão se associou positivamente a excesso de atendimento nas UAPS. De maneira geral, as mulheres buscam mais os serviços de saúde do que os homens, e esse diferencial pode estar relacionado às variações no perfil de necessidades de saúde entre os gêneros, entre elas a gravidez e o parto e ainda o maior interesse das mulheres com relação à sua saúde ${ }^{30,31}$.

Vale enfatizar que, pela possibilidade de apresentar comorbidades físicas e/ou mentais, a depressão nem sempre é detectada ou tem tratamento adequado. A dificuldade do não reconhecimento dos transtornos mentais está na probabilidade de esses pacientes relatarem apenas sintomas somáticos, ao procurar os serviços de saúde. Nesse contexto, um estudo ${ }^{32}$ sugere que os sintomas somáticos tendem a mascarar transtornos de ordem psicológica/psiquiátrica.

Apesar de existirem vários instrumentos de detecção, a depressão tem sido subdiagnosticada, pois, no Brasil, ainda não há a rotina de inclusão de instrumentos de rastreamento nos serviços de atenção primária9,33,34. A constatação de que 53\% das mulheres que apresentaram escore maior ou igual 10 no PHQ-9 não relataram ter apresentado ou ter sido diagnosticadas com depressão alerta para a importância desse rastreamento.

A lacuna na utilização de instrumentos de rastreamento de depressão na atenção primária pode estar vinculada à não capacitação dos profissionais e/ou ao reduzido tempo das consultas. Os profissionais ainda estão focados especialmente na queixa do paciente e nem sempre consideram seu contexto de vida, enfatizando uma ação limitada e ineficaz dos serviços de saúde, que também não se articulam/integram com os serviços especializados em saúde mental. Por outro lado, é preciso considerar também que os pacientes 
podem ter preconceito em relação ao diagnóstico de depressão e descrença quanto ao tratamento.

$\mathrm{Na}$ análise multivariada, permaneceram como fatores associados à ocorrência de depressão na população estudada: possuir baixa escolaridade, trabalhar atualmente e ter doença mental prévia (esquizofrenia, transtorno bipolar, psicoses). Como fatores de proteção observaram-se: viver com companheiro, realizar atividades físicas regularmente e relatar autoavaliação positiva de saúde.

Quanto à escolaridade, verificou-se uma relação inversa com a ocorrência do desfecho, isto é, quanto menor escolaridade, maior a probabilidade de ocorrência de depressão. Estudos relatam que a escolaridade e a pobreza podem ser um determinante dos índices de depressão, pois eles estão associados a condições sociais como desemprego, baixa qualidade de moradia e alimentação inadequada ${ }^{20,30}$. Isso reforça a hipótese de que as condições de vida, acompanhadas de condições sociais impróprias, sejam determinantes no aparecimento das doenças, pois levam a um estado de desesperança que favorece a incapacidade para lidar com situações estressoras e acontecimentos adversos e frustrantes ${ }^{30}$. A escolaridade aumenta a possibilidade de escolhas na vida, além de influenciar na autoestima, motivando atitudes e comportamentos mais saudáveis, e sua falta diminui o poder de decisão do indivíduo, gerando incapacidade de influenciar o meio, resultando em comprometimento da saúde ${ }^{35}$.

Observou-se que estar trabalhando atualmente mostrou associação com o desfecho depressão. Esse achado pode ser explicado em decorrência de que o "ambiente de trabalho vem influenciando consideravelmente na vida dos indivíduos, especialmente porque é no trabalho que ele passa a maior parte de seu tempo e onde estabelece grande parte de seus vínculos sociais" ${ }^{\prime \prime 6}$. Por isso, esse ambiente passou a ter alta relevância na formação e manutenção da identidade, consistindo num local importante para o sentimento de valorização pessoal, tornando-se propício para conflitos e o aparecimento de patologias, sendo a mais comum delas a depressão ${ }^{37}$.

Além disso, nas últimas décadas, houve uma mudança no papel da mulher na família e na sociedade, sem um reconhecimento adequado de seus efeitos, mantendo uma invisibilidade das atividades realizadas e centralizando-as como atribuição feminina ${ }^{38}$. A mulher tem sido solicitada a contribuir financeiramente no sustento da família e ainda continuar com as responsabilidades do lar, maternidade, cobranças sociais, o que acarreta sobrecarga de trabalho. Enfim, é possível afirmar que a exposição a condições de trabalho adversas é fator de risco para a saúde ${ }^{36}$.

Os achados também assinalaram a presença de outros transtornos mentais (esquizofrenia, transtorno bipolar, psicoses) como risco para a depressão. Uma das maiores dificuldades na abordagem do paciente com comorbidades mentais diz respeito ao fato de que o diagnóstico primário muitas vezes é difícil de ser estabelecido inicialmente. A não identificação de uma comorbidade implicará resposta parcial ao tratamento ${ }^{39}$. No campo da atenção primária, isso se torna mais relevante ao considerar que não há especialistas na área e não são usados rotineiramente instrumentos de avaliação para a detecção dos transtornos mentais ${ }^{40}$. Estudos mostram que a melhora na detecção de tais transtornos no nível primário pode incidir em diminuição na procura por atendimento médico e na utilização de recursos em saúde, o que contribuiria para desonerar o sistema de saúde ${ }^{41,42}$.

A inatividade física é um fator que tem se associado fortemente a estados variados da depressão, o que se coaduna com os resultados aqui obtidos. O bem-estar que os exercícios físicos geram melhora o condicionamento físico, além de promover benefícios psicofisiológicos em curto prazo, evidenciando que a prática é de relevante importância tanto como prevenção quanto para o tratamento da depressão ${ }^{42}$.

Outro fator de proteção relacionado a depressão encontrado neste estudo foi ser casada ou viver com companheiro, já identificado em outros estudos ${ }^{22,43}$. Diversas hipóteses tentam explicar esse efeito protetor como, por exemplo, o casamento pode levar a hábitos de vida mais saudáveis, além de oferecer um suporte familiar que colabora no cuidado e na resolução das situações de dificuldade vivenciadas no dia a dia. Assim sendo, quanto maior a proximidade com os elementos com quem se estabelece relações interpessoais íntimas, menor é a vulnerabilidade do indivíduo à depressão. Estudo verificou que estar sozinho aumenta em até oito vezes a probabilidade de desenvolver sinais de depressão ${ }^{44}$.

É importante enfatizar que a avaliação do estado de saúde consiste na percepção que os indivíduos possuem de sua própria saúde, sendo um indicador que engloba tanto componentes físicos quanto emocionais desses indivíduos, além de aspectos do bem-estar e da satisfação com a própria vida ${ }^{45}$. Boa saúde pode ser interpretada distintamente, em termos de ausência ou presença de um problema de saúde, de funcionalidade física geral ou de padrões culturais de comportamento saudável. A avaliação subjetiva das pessoas sobre a sua própria condição de saúde é um indicador importante do impacto da doença sobre o bem-estar individual. Os processos interpretativos dos sujeitos ultrapassam a saúde física, derivando-se de sua maneira de ver a vida, da presença de redes sociais, passando, portanto, por dimensões psicológicas e socioculturais ${ }^{9,46}$. No presente estudo, a autoavaliação positiva se apresenta como um fator de proteção contra a depressão.

Algumas limitações deste estudo podem estar relacionadas à utilização de medidas de autorrelato sem confirmação clínica. Foi utilizado um instrumento de rastreamento (PHQ-9) que aponta os indivíduos em maior risco de estar apresentando episódio depressivo maior. O diagnóstico definitivo da doença somente poderá ser firmado por meio da consulta com profissionais de saúde mental. 
Destaca-se que o PHQ-9 teve sua validade testada em vários níveis de atenção à saúde em diversas línguas e contextos culturais. Foi também utilizado em outros estudos internacionais como o realizado com estudantes universitários na Nigéria ${ }^{47}$, com média de idade de 24 anos, a maioria homens, encontrando o valor maior ou igual a 10 como o melhor ponto de corte para rastreamento de transtorno depressivo maior, apresentando sensibilidade nesse ponto de $84,6 \%$ e especificidade de 99,4\%, superiores às encontradas em Pelotas. Em estudo realizado com puérperas nos Estados Unidos, comparou-se a utilização do ponto de corte maior ou igual a 10 com a utilização do algoritmo, constatando-se valores mais altos de sensibilidade e especificidade para o primeiro ${ }^{48}$.

Não se pode desconsiderar também que o delineamento transversal pode implicar causalidade reversa entre o desfecho e as variáveis de exposição situação conjugal e situação de trabalho.

\section{CONCLUSÃO}

Os resultados deste estudo revelam prevalência de depressão de 19,7\% nas mulheres de 20 a 59 anos de áreas cobertas pela Estratégia de Saúde da Família, apontando para a necessidade de um cuidado especial na atenção primária à saúde às mulheres com baixa escolaridade, que trabalham, apresentam doença mental e não praticam exercícios físicos, visando derivar em benefícios consideráveis para a redução do sofrimento e promoção de saúde.

O cuidado à saúde mental é uma questão ainda negligenciada, principalmente na atenção primária, o que repercute na inviabilização da construção de uma rede de atenção articulada à rede de saúde em geral e também a outros recursos intersetoriais que garantiriam a integralidade das ações de saúde.

Cabe aos profissionais da saúde aprimorar suas práticas, valorizando as formas de investigação da depressão e dos possíveis fatores associados, considerando as particularidades e o contexto biopsicossocial e cultural de cada indivíduo e fornecendo o cuidado integral, que vai além da queixa-conduta, contribuindo para a equidade no sistema de saúde brasileiro.

Espera-se que com este estudo se possa contribuir para o entendimento dos determinantes da depressão e, desse modo, fornecer evidências para a formulação de políticas públicas que contemplem a saúde mental no nível primário de atenção.

\section{CONTRIBUIÇÕES INDIVIDUAIS}

Todos os autores contribuíram significativamente na concepção e desenho do estudo, análise, interpretação dos da- dos, bem como na elaboração do artigo e na aprovação da versão final a ser publicada.

\section{CONFLITOS DE INTERESSE}

Os autores não possuem conflitos de interesse a serem declarados.

\section{REFERÊNCIAS}

1. American Psychiatric Association (APA). Diagnostic and Statistical Manual of Mental disorders - DSM-5. 5th ed. Washington: APA; 2013.

2. Boing AF, Melo GR, Boing AC, Moretti-Pires RO, Peres KG, Peres MA. Associação entre depressão e doenças crônicas: um estudo populacional. Rev. Saúde Pública. 2012;46(4):617-23.

3. World Health Organization (WHO). Depression and Other Common Mental Disorders - Global Health Estimates. 2017. Disponivel em: http://apps.who.int/iris/bitstream/10665/254610/1/ WH0-MSD-MER-2017.2-eng.pdf?ua=1. Acesso em: 22 ago. 2017.

4. Costa TS. Rastreamento de sintomas depressivos em usuários assistidos pela Estratégia de Saúde da Família em um município de pequeno porte no nordeste brasileiro [tese]. São Paulo: Faculdade de Ciências Médicas da Santa Casa de São Paulo; 2015.

5. Apóstolo JLA, Figueiredo MH, Mendes AC, Rodrigues MA. Depressão, ansiedade e estresse em usuários de cuidados primários de saúde. Rev Latino-Am Enfermagem. 2011;19(2).

6. Grandes G1, Montoya I, Arietaleanizbeaskoa MS, Arce V, Sanchez A; MAS Group. The burden of mental disorders in primary care. Eur Psychiatry. 2011;26(7):428-35.

7. Lopez MRA, Ribeiro JP, Ores LC, Jansen K, Souza LDM, Pinheiro RT, et al. Depressão e qualidade de vida em jovens de 18 a 24 anos no sul do Brasil. Rev Psiquiatr Rio Gd Sul. 2011;33(2):103-8.

8. Gabilondo A, Vilagut G, Pinto-Meza A, Haro JM, Alonso J. Comorbidity of major depressive episode and chronic physical conditions in Spain, a country with low prevalence of depression. Gen Hosp Psychiatry. 2012;34(5):510-7.

9. Molina MRAL, Wiener CD, Branco JC, Jansen K, Souza LDM, Tomasi E, et al. Prevalência de depressão em usuários de unidades de atenção primária. Rev Psiquiatr Clín. 2012;39(6):194-7.

10. Rancans E, Vrublevska J, Snikere S, Koroleva I, Trapencieris M. The point prevalence of depression and associated sociodemographic correlates in the general population of Latvia. J Affect Disord. 2014;156:104-10.

11. Sacks RM, Greene J2, Hibbard JH3, Overton V. How well do patient activation scores predict depression outcomes one year later? J Affect Disord. 2014;169:1-6.

12. Lopes CS, Hellwig N, Silva GA, Menezes PR. Inequities in access to depression treatment: results of the Brazilian National Health Survey - PNS. Int J Equity Health. 2016;15:154.

13. Loosli L, Pizeta FA, Loureiro SR. Escolares que convivem com a depressão materna recorrente: diferenças entre os sexos. Psic Teor Pesq. 2016;32(3):1-10.

14. Santos IS, Tavares BF, Munhoz TN, Almeida LS, Silva NT, Tams BD, et al. [Sensitivity and specificity of the Patient Health Questionnaire-9 (PHQ-9) among adults from the general population]. Cad Saude Publica. 2013;29(8):1533-43.

15. Kroenke K, Spitzer RL, Williams JB. The PHQ-9: validity of a brief depression severity measure. J Gen Intern Med. 2001;16(9):606-13.

16. Instituto Brasileiro de Geografia e Estatística (IBGE). Pesquisa Nacional de Saúde 2013: percepção do estado da saúde, estilos de vida e doenças crônicas - Brasil, Grandes Regiões e Unidades da Federação. 2014. Disponível em: ftp://ftp.ibge.gov.br/PNS/2013/pns2013. pdf. Acesso em: 10 abr. 2016.

17. Szwarcwald CL, Malta DC, Pereira CA, Vieira MLFP, Conde WL, Souza Júnior PRB, et al. Pesquisa Nacional de Saúde no Brasil: concepção e metodologia de aplicação. Ciênc Saúde Coletiva. 2014;19(2):333-42.

18. DSM-IV-TRTM - Manual diagnóstico e estatístico de transtornos mentais. Tradução: Cláudia Dornelles. $4^{a}$ ed., rev. Porto Alegre: Artmed; 2002. 
19. Rombaldi AJ, Silva MC, Gazalle FK, Azevedo MR, Hallal PC. Prevalência e fatores associados a sintomas depressivos em adultos do sul do Brasil: estudo transversal de base populacional. Rev Bras Epidemiol. 2010;13(4):620-9.

20. Stopa SR, Malta DC, Oliveira MM, Lopes CS, Menezes PR, Kinoshita RT. Prevalência do autorrelato de depressão no Brasil: resultados da Pesquisa Nacional de Saúde, 2013. Rev Bras Epidemiol. 2015;18(Supl 2):170-80.

21. Silva MT, Galvão TF, Martins SS, Pereira MG. Prevalence of depression morbidity among Brazilian adults: a systematic review and meta-analysis. Rev Bras Psiquiatr. 2014;36(3):262-70

22. Munhoz TN. Prevalência e fatores associados à depressão em adultos: estudo de base populacional [dissertação]. Pelotas: Universidade Federal de Pelotas; 2012.

23. Laguardia J. Raça e epidemiologia: as estratégias para construção de diferenças biológicas. Ciênc Saúde Coletiva. 2007;12(1):253-61.

24. Máximo GC. Aspectos sociodemográficos da depressão e utilização de serviços de saúde no Brasil [tese]. Belo Horizonte: Universidade Federal de Minas Gerais; 2010.

25. Moreira-Almeida A, Lotufo Neto F, Koenig HG. Religiousness and mental health: a review. Rev Bras Psiquiatr. 2006;28(3):242-50.

26. Rondina RC, Gorayeb R, Botelho C. Características psicológicas associadas ao comportamento de fumar tabaco. J Bras Pneumol. 2007;33(5):592-601.

27. Stein MB, Mellman TA. Anxiety disorders. In: Kryger MH, Roth T, Dement WC, editors. Principles and practice of sleep medicine. 4th ed. Philadelphia: WB Saunders; 2005. p. 1297-311.

28. Chellappa SL, Araujo JF. Transtornos do sono em pacientes ambulatoriais com depressão. Rev Psiquiatr Clín. 2006;33(5):233-8.

29. Carvalho IPA, Carvalho CGX, Lopes JMC. Prevalência de hiperutilizadores de serviços de saúde com histórico positivo para depressão em Atenção Primária à Saúde. Rev Bras Med Fam Comunidade. 2015;10(34):1-7.

30. Gomes R, Nascimento EF, Araujo FC. Por que os homens buscam menos os serviços de saúde do que as mulheres? As explicações de homens com baixa escolaridade e homens com ensino superior. Cad Saúde Pública. 2007;23(3):565-74.

31. Pinheiro RS, Viacava F, Travassos C, Brito AS. Gênero, morbidade, acesso e utilização de serviços de saúde no Brasil. Ciênc Saúde Coletiva. 2002;7(4):687-707.

32. Skapinakis P, Araya R. Common somatic symptoms, causal attributions of somatic symptoms and psychiatric morbidity in a cross-sectional community study in Santiago, Chile. BMC Res Notes. 2011;4:155.

33. Arantes DV. Depressão na atenção primária à saúde. Rev Bras Med Fam Comunidade. 2007;2(8):261-70.
34. Corso AN, Costa LS, Fleck MPA, Heldt E. Impacto de sintomas depressivos na qualidade de vida de usuários da rede básica de saúde. Rev Gaúcha Enfermagem. 2009,30(2):257-62.

35. Castan JU, Brentano V. Psicodiagnóstico na Unidade de Internação Psiquiátrica de um Hospital Universitário: descrição da demanda de 2015. Rev SBPH. 2017;20(1):195-208.

36. Hämmig 0, Bauer GF. Work, work-life conflict and health in an industrial work environment. Occup Med (Lond). 2014;64(1):34-8.

37. Moreira V, Maciel RH, Araujo TQ. Depressão: os sentidos do trabalho. Rev NUFEN. 2013;5(1):45-56

38. Araújo TM, Pinho PS, Almeida MMG. Prevalência de transtornos mentais comuns em mulheres e sua relação com as características sociodemográficas e o trabalho doméstico. Rev Bras Saúde Matern Infant. 2005;5(3):337-48.

39. Sanches RF, Assunção S, Hetem LAB. Impacto da comorbidade no diagnóstico e tratamento do transtorno bipolar. Rev Psiquiatr Clín. 2005;32(Supl 1):71-7.

40. Bolsoni LM, Zuardi AW. Estudos psicométricos de instrumentos breves de rastreio para múltiplos transtornos mentais. J Bras Psiquiatr. 2015;64(1):63-9.

41. Gonçalves DM, Kapczinski F. Prevalência de transtornos mentais em indivíduos de uma unidade de referência para Programa Saúde da Família em Santa Cruz do Sul, Rio Grande do Sul, Brasil. Cad Saúde Pública. 2008;24(9):2043-53.

42. Barros MBA, Lima MG, Azevedo RCS, Medina LBP, Lopes CS, Menezes PR, et al. Depressão e comportamentos de saúde em adultos brasileiros - PNS 2013. Rev Saúde Pública. 2017;51(Supl 1).

43. Andrade L, Caraveo-Anduaga JJ, Berglund P, Bijl RV, De Graaf R, Vollebergh W, et al. The epidemiology of major depressive episodes: results from the International Consortium of Psychiatric Epidemiology (ICPE) Surveys. Int J Methods Psychiatr Res. 2003;12(1):3-21.

44. Monteiro IS. 0 contributo das experiências familiares, vinculação e apoio social para a depressão no adulto [tese]. Porto: Universidade do Minho; 2010.

45. Pavão ALB, Werneck GL, Campos MR. Autoavaliação do estado de saúde e a associação com fatores sociodemográficos, hábitos de vida e morbidade na população: um inquérito nacional. Cad Saúde Pública. 2013;29(4):723-34

46. Horwitz AV, Wakefield JC. A tristeza perdida - como a psiquiatria transformou a depressão em moda. São Paulo: Summus; 2010

47. Adewuya A0, Ola BA, Afolabi 00. Validity of the patient health questionnaire (PHQ-9) as a screening tool for depression amongst Nigerian university students. J Affect Disord. 2006;96(1-2):89-93.

48. Gjerdingen D, Crow S, McGovern P, Miner M, Center B. Postpartum depression screening at well-child visits: validity of a 2-question screen and the PHQ-9. Ann Fam Med. 2009;7(1):63-70. 